\title{
Mixing measures: testing an assumption of the attention network test
}

\author{
Meghan M. McConnell • David I. Shore
}

Published online: 15 January 2011

(C) Psychonomic Society, Inc. 2011

\begin{abstract}
The human attention system has been subdivided into three networks that appear to be functionally and anatomically independent: alerting, orienting, and executive control. The Attention Network Test (ANT) is a quick and easy tool that measures the efficiency of these three networks by averaging reaction time and accuracy scores across several different cue and flanker conditions. Using ANOVAs and correlation procedures, we found that (a) intranetwork correlations were surprisingly low, and (b) Cue X Flanker interactions were found within alerting, orienting, and executive control measures. Taken together, these findings highlight the interaction between the three networks and the potential difficulty in accurately measuring them with this task.
\end{abstract}

Keywords Attention networks - Attention Network Test . Alerting · Orienting · Executive Function

Attention manifests itself in almost every aspect of human behavior, ranging from basic perceptual skills to complex cognitive abilities. As a result, attention is one of the oldest topics in experimental psychology. Attention was initially viewed as a general mechanism that influenced the brain as a whole, but recent neuropsychological and neuroimaging studies have demonstrated that attention is a multidimensional construct made up of at least three separate networks: alerting, orienting, and executive control (Fan, McCandliss, Fossella, Flombaum \& Posner, 2005; Fan, McCandliss, Sommer, Raz, \& Posner, 2002; Posner \& Fan, 2004; Posner $\&$ Peterson, 1990). The alerting network maintains a state of vigilance in preparation for upcoming stimuli. The orienting network is responsible for the movement of

M. M. McConnell • D. I. Shore $(\square)$

Department of Psychology, Neuroscience \& Behaviour,

McMaster University,

Hamilton, ON, Canada L8S 4K1

e-mail: dshore@mcmaster.ca attention throughout the environment in order to attend to incoming sensory information. And, finally, the executive control network enables conflict resolution, error detection, and inhibitory control. The Attention Network Test (ANT) is a quick and easy tool that measures the efficiency of these three networks by averaging reaction time (RT) and accuracy scores across several different cue and flanker conditions. The purpose of the present study was to assess the appropriateness of averaging across cue and flanker conditions to obtain separate measures of the three attention networks.

These three subsystems correspond to discrete anatomical and neurochemical circuits that maintain a degree of functional independence. The alerting response is associated with activity in the frontal and parietal regions (Coull, Frith, Frackowiak, \& Grasby, 1996; Coull, Nobre, \& Frith, 2001) and relies on noradrenalin in the locus coeruleus and the parietal cortex (Sturm \& Willmes, 2001). Several noradrenergic genes (i.e., MAOA, $\mathrm{D} \beta \mathrm{H}$ ) have been linked to the efficiency of the alerting network (Bellgrove, Hawi, Gill, \& Robertson, 2006; Fossella et al., 2002). The orienting network is associated with the superior parietal lobe, superior colliculus, and frontal eye fields (Corbetta, 1998; Corbetta, Kincade, Ollinger, McAvoy, \& Shulman, 2000; Parasuraman, Greenwood, Haxby, \& Grady, 1992). Orienting efficiency has been linked to the cholinergic system stemming from posterior brain regions (Davidson, Cutrell, \& Marrocco, 1999; Greenwood, Fossella, \& Parasuraman, 2005). Accordingly, polymorphisms in genes associated with the cholinergic system (i.e, CHRNA4, APOE gene) influence the ability to orient attention to perceptual cues (Parasuraman, Greenwood, Kumar, \& Fossella, 2005). Finally, executive control relies on the activation of the anterior cingulate cortex (ACC) as well as on the lateral prefrontal cortex (Bush, Luu, \& Posner, 2000; Etkin, Egner, Peraza, Kandel, \& Hirsch, 2006; MacDonald, Cohen, Stenger, \& Carter, 2000). These two neural areas are rich in dopamine receptors, suggesting that the dopaminergic system is a critical component of the 
executive control network (Bush et al., 2000). Polymorphisms in dopamine receptors (DRD4), transporters (DAT1), and enzymes (COMT) are able to account for variability in tasks that tap into various executive control (Diamond, Briand, Fossella, \& Gehlbach, 2004; Fossella et al., 2002). Thus, there is good evidence that these three attention constructs have separate instantiations in the brain.

A recent experimental paradigm called ANT was developed to measure the efficiency of the three attention networks simultaneously and to assess their interrelations (Fan, McCandliss, Sommer, et al., 2002). The ANT combines multiple warning cues and target flanker displays to obtain measures of the three attention networks in a single task (see Fig. 1). In each ANT trial, participants are shown a row of five arrow stimuli and are required to report the direction of a central arrow (left or right). The target arrow is surrounded by distracting flanker arrows that can point in the same direction as the central arrow (congruent), in the opposite direction (incongruent), or in neither direction (straight lines; neutral condition). The arrow display can appear either above or below fixation. Therefore, correct identification of the target direction requires participants not only to inhibit potentially irrelevant information provided by the flanking arrows but also to shift attention either upward or downward to the target location.

The arrow display is preceded by one of four cues. In the no cue condition, participants receive no warning before the target display appears. In the cued conditions, a cue is presented at the central fixation location (center cue condition), above or below fixation at which the arrow display is to appear (spatial cue condition), or both above and below fixation (double cue condition). The combination of the flanker (congruent, incongruent, or neutral) and cue (center cue, double cue, spatially informative cue, or no cue) conditions allows researchers to extract measures of alerting, orienting, and executive control through a series of orthogonal contrasts between mean correct RTs.

The alerting score is calculated by subtracting the mean RT in the double cue condition from the mean RT in the no cue condition, regardless of the type of flanker stimuli. The logic is that the appearance of the double cue alerts participants to the upcoming onset of the target display, and because such a warning is not present in the no cue condition, the RT difference between these two conditions should provide a measure of alerting efficiency.

The orienting network score is calculated by subtracting the mean RT in the spatial cue condition from the mean RT in the central cue condition, regardless of the flanker condition. In this instance, the spatial cue informs the participant of the precise location of the target stimulus, whereas the center cue does not. Therefore, when presented with a spatial cue, participants are able to orient their attention to the target location prior to the appearance of the target. As a result, the
RT difference between the central and spatial cue conditions should provide a measure of orienting efficiency.

Finally, the executive control score is calculated by subtracting the mean RT in the congruent condition from the mean RT in the incongruent condition, which provides a measure of conflict resolution. When presented with an incongruent target display, participants must deal with the conflicting information provided simultaneously by the target and flanker arrows. On the other hand, during congruent target displays, the flanker and target arrows provide participants with the same information about the target response; thus, no conflict resolution is required. Therefore, the flanker interference effect- that is, the RT difference between the congruent and incongruent arrow displays - provides a measure of executive control.

One of the key assumptions of the ANT is that the three components of attention operate independently of one another. In the original report of the ANT, Fan, McCandliss, Sommer, et al. (2002) found support for independence between networks. They observed no correlations between scores obtained for each network. They did, however, report a significant interaction between cue condition and flanker type, which implies that attention networks are not entirely independent (Fan, McCandliss, Sommer, et al., 2002). In order to investigate potential interactions across attention networks, Callejas and colleagues (Callejas, Lupiáñez, Funes, \& Tudela, 2005; Callejas, Lupiáñez, \& Tudela, 2004) modified Fan's task by including a new alerting stimulus: a short-duration high-frequency tone. This modification enabled the researchers to measure each network independently and to quantify the effect of one network on the other two networks. In a series of experiments, Callejas et al. reported (a) a larger flanker-congruency effect after an alerting stimulus, suggesting that the executive control network is inhibited by the alerting network, (b) a smaller congruency effect on cued versus uncued trials, demonstrating that the orienting network enhances the efficiency of executive control, and (c) a larger cuing effect following an alerting signal, suggesting that the alerting network speeds up the efficiency of the orienting network (Callejas, Lupiáñez, Funes, et al., 2005; Callejas, Lupiáñez, \& Tudela, 2004). These findings have since been replicated using other versions of the ANT (ANT-R; Fan, Gu, et al., 2009).

Although the interactive nature between attention network scores, as measured by the ANT, is well documented in the literature, researchers have failed to examine potential interactions within each network. For example, in the original ANT, executive control was calculated by collapsing across the four cue conditions, whereas the alerting and orienting scores were calculated by collapsing across the three flanker conditions. Because previous research has clearly demonstrated that the networks interact (cf. Callejas, Lupiáñez, Funes, et al., 2005; Callejas, Lupiáñez, \& Tudela, 2004; Fan, Gu, et al., 2009), we 
Fig. 1 Attention network test (ANT) design and procedure: (a)

The four waning cue conditions.

(b) The three flanker conditions.

(c) The sequence of events for a given trial. The participants' task was to determine whether the central arrow in the flanker display was pointing to the left or right. The target display was presented equally above (as shown here) or below central fixation $(+)$. The following subtractions were used to calculate the efficiency of the three attention networks. Alerting: Trimmed mean reaction time (RT) for no cues - trimmed mean RT for double cues. Orienting: Trimmed mean RT for central cuestrimmed mean RT for spatial cues. Executive function:

Trimmed mean RT for incongruent flankers - trimmed mean RT for congruent flankers
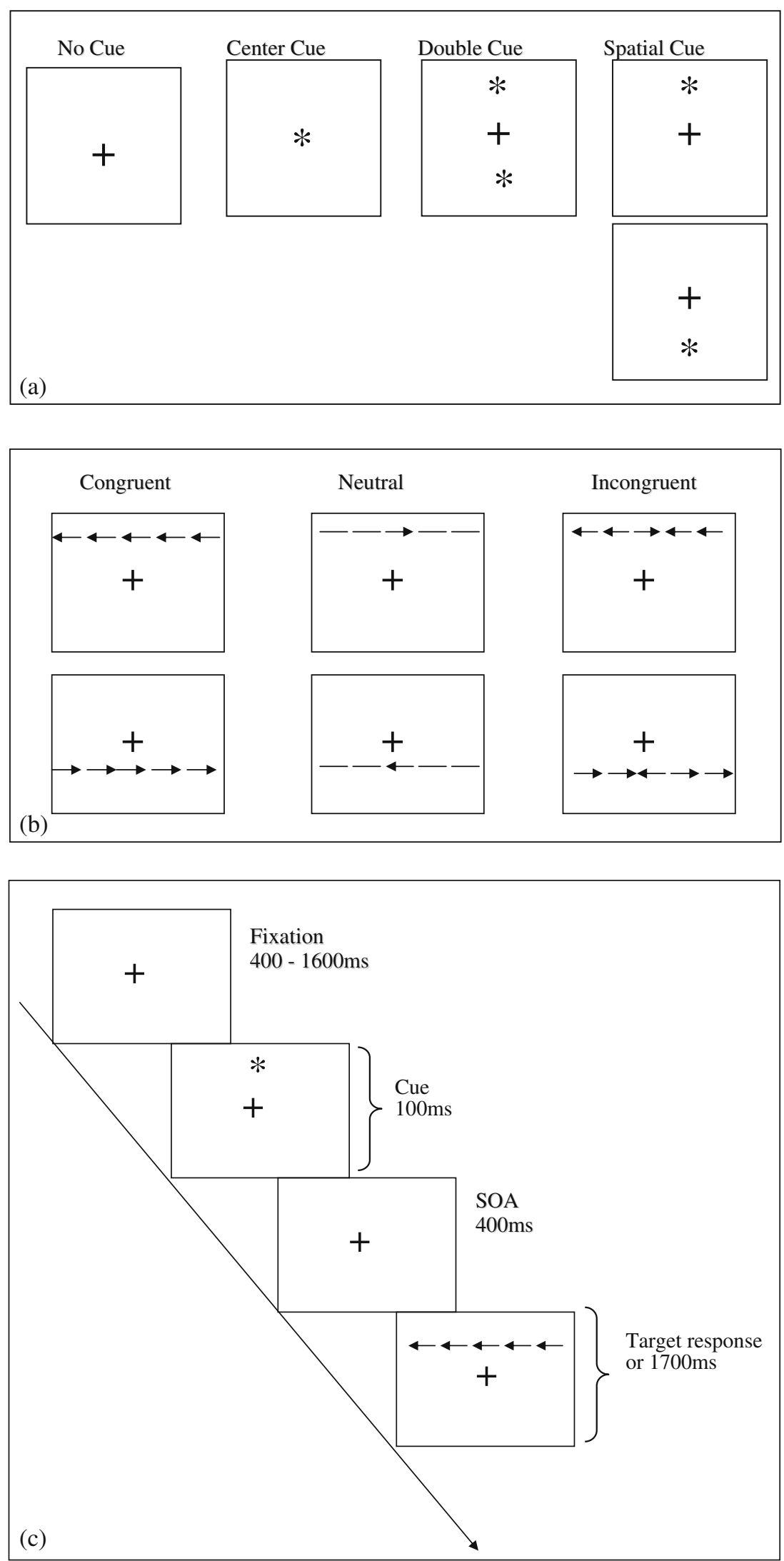
thought it was important to determine whether there were any interactions across the individual cue and flanker conditions within each network.

Therefore, the purpose of the present study was to assess the appropriateness of averaging across cue and flanker conditions to obtain separate measures of the three attention networks. We used ANOVAs and correlation procedures, which allowed us to examine intranetwork differences and correlations. The ANT assumes that the collapsed components are all measuring the same attentional process. For example, we obtain a single measure of executive control by collapsing across four separate measures: executive control on trials that contained no cue, executive control on trials that contained a central cue, executive control on trials that contained a double cue, and executive control on trials that contained a spatial cue. In order to justify collapsing across these four cue conditions to get a single measure of executive control, we must assume that all four components of executive control are providing reliable measures of the same underlying processes. If this assumption is valid, we would expect similar executive control scores across the four cue conditions and similar alerting and orienting scores across the three flanker conditions. Furthermore, we would expect the individual cue and flanker combinations that compose each network to be correlated with one another (i.e., all collapsed components are measuring the same underlying process and therefore should have similar values). More specifically, we would expect the correlations within each network to be greater than the correlations between the three networks.

However, given that Callejas, Lupiáñez, Funes, et al. (2004), Callejas, Lupiáñez, and Tudela (2005), and Fan, Gu, et al. (2009) have found that different levels of alerting and orienting can inhibit or enhance executive control, respectively, it is reasonable for one to assume that the magnitude of flanker-congruency effects may vary depending on the identity of the preceding cue. In this case, we may find significant differences in the mean values of the different Cue X Flanker combinations and low correlations between the intranetwork composites. We used ANOVAs and correlation procedures to examine intranetwork differences and correlations. If the intranetwork composites are providing reliable measures of alerting, orienting, and executive control, we would expect (a) little differences in the mean values of the different Cue X Flanker combinations, and (b) high correlations between the intranetwork composites.

\section{Method}

Participants

A total of 588 undergraduate students from McMaster University participated for course credit. These participants ran in the ANT in three unrelated experiments. Each experiment used a between-subjects design. The first experiment included 273 participants (mean age $=19.1$ years, 64 males, 22 left-handed) and was designed to investigate daily and yearly temporal variations in the three attention networks (McConnell \& Shore, in prep). To do this, the ANT was administered up to three times a day $(0900 \mathrm{~h}, 1230 \mathrm{~h}$, or $1630 \mathrm{~h}$ ) to different participants each time, Monday to Friday from September 2006 to April 2007. From this experiment, we found that (a) the flanker interference effect was greater (suggesting less efficient executive control) in the first semester than in the second semester, and that (b) alerting measures were greater in the last 4 weeks than in the first 4 weeks of the term.

In the second experiment, we examined whether changes to and from daylight savings time (DST) influenced the efficiency of attention networks and included 249 participants (mean age $=19.2$ years, 69 males, 21 left-handed; McConnell \& Shore, submitted). In this experiment, we administered the ANT to different participants on the Mondays preceding and following the change to DST in the spring and after the return to standard time in the fall. We found that the orienting network was detrimentally affected after both changes.

Finally, in the third experiment, we studied the effects of music-induced mood changes on attention network scores and collected data from 66 participants (mean age = 19.3 years, 16 males, four left-handed; McConnell \& Shore, in press). In this experiment, we manipulated emotional valence (positive vs. negative) and arousal (high vs. low) by having participants listen to one of four versions of Mozart's sonata K. 448 that varied in mode (major or minor) and tempo (fast or slow). This music was originally used in an experiment conducted by Husain, Thompson, and Schellenberg (2002), who set out to investigate whether musical mode and tempo could modulate measures of spatial abilities. Participants listened to the musical excerpt for $10 \mathrm{~min}$ prior to the administration of the ANT. The results from this experiment revealed that emotional valence and arousal influenced measures of executive control, but not on alerting or orienting. Individuals who experienced positive emotional valence had less efficient control over their responses than did those who experienced negative emotional valence, but only when arousal levels were high. Positive and negative valence did not influence executive control measures when arousal levels were low.

In total, the present experiment included data from 588 subjects; however, 10 participants (eight from the first experiment and two from the third experiment) were excluded for having a mean error rate greater than $35 \%$. All of the participants were enrolled in first- or second-year psychology courses and reported normal or corrected-to- 
normal vision. The McMaster Research Ethics Board approved the experimental design, and written informed consent was obtained from each participant.

\section{Materials}

In the present study, we used the same version of the ANT first presented by Fan, McCandliss, Somer, et al. (2002; see Fig. 1). All of the stimuli were presented on a white background using MATLAB software and the Psychophysics Toolbox (Brainard, 1997). The target display consisted of a central arrow, pointing either to the left or to the right, and four flanking stimuli that could be either straight lines or arrows pointing in the same direction as, or opposite direction of, the target arrows. Each arrow stimulus subtended approximately $1.6^{\circ}$ of visual angle and was presented approximately $1.0^{\circ}$ of visual angle above or below fixation in equal proportions.

\section{Procedure}

Participants in Experiments 1 and 3 were seated individually in a dimly lit room approximately $50 \mathrm{~cm}$ away from a 17 -in. computer monitor. Participants in Experiment 2 were tested in groups ranging from two to 13 individuals who were seated in a naturally lit room approximately $50 \mathrm{~cm}$ away from a 21 -in. computer monitor.

Each experimental session included one block of 24 practice trials and three blocks of 96 experimental trials. During the practice trials, but not during the experimental trials, participants received feedback on their accuracy.

The sequence of events for a given trial can be seen in Fig. 1. Each trial began with the presentation of a fixation point (a plus sign) for a variable duration $(400-1,600 \mathrm{~ms})$. The fixation point was followed by one of four cue conditions that consisted of asterisks that were equally likely to appear at fixation (center cue), above and below fixation (double cue), in the same location as the upcoming target (spatial cue), or not at all (see Fig. 1a). Each cue was equally likely to appear and remained on the screen for $100 \mathrm{~ms}$. After a 400 -ms presentation of the fixation display, the target and flankers appeared either above or below the fixation point. The participant was to respond to the direction of the central arrow as quickly and accurately as possible by pressing the " $\mathrm{z}$ " key if the target arrow was pointing to the left or the " $m$ " key if the target was pointing to the right. On one-third of the trials, the target and flanking arrows pointed in the same direction (congruent trials), and on another third of the trials, the target arrow pointed in a direction opposite from the four flanking arrows (incongruent trials). The remaining trials contained the target arrow flanked by four straight lines (Fig. 1b). The target display subsequently appeared and remained onscreen until a response was made, to a maximum of $1,700 \mathrm{~ms}$. After participants made their responses (or after $1,700 \mathrm{~ms}$, if no response was made), the target display disappeared while the fixation point remained onscreen for a duration that depended on the duration of the first fixation period minus the participants' RTs. As a result, all trials had the same duration $(3,500 \mathrm{~ms})$.

\section{Data analysis}

Correct RTs were subjected to a trimmed mean procedure. For each participant, the top and bottom $10 \%$ of the data were removed from each of the 12 factorial cells (three flanker and four cue conditions) of the RT data after error trials were removed. ${ }^{1}$ We chose to use the trimmed mean as our measure of central tendency because it is less susceptible than the arithmetic mean to the effects of outliers and violations in normality and heteroscedasticity (see MacLeod et al., 2010). Classic parametric statistics are sensitive to the presence of outliers and to deviations from normality and homoscedasticity, and ignoring these data characteristics can lead to erroneous results (for review, see Erceg-Hurn \& Mirosevich, 2008). ${ }^{2}$

Measures of alerting, orienting, and executive control were calculated by making orthogonal comparisons across trimmed mean RTs for different cue and flanker conditions. These difference scores were used in place of mean trimmed RTs. Alerting scores were calculated by subtracting the trimmed mean RTs for double cue trials from the trimmed mean RTs for the no cue trials for each of the three flanker conditions (neutral, congruent, and incongruent). Orienting efficiency was measured by subtracting the trimmed mean RTs for spatial cue trials from central cue trials for neutral, congruent, and incongruent flanker displays. And, finally, executive control scores were obtained by subtracting trimmed mean RTs for congruent trials from the trimmed mean RT for incongruent trials for each of the four cue conditions (no cue, double cue, central cue, spatial cue).

Trimmed mean RTs and mean error rates were assessed using a 3 (flanker type) X 4 (cue type) repeated measures ANOVA. Individual ANOVAs were also calculated for each of the attention networks. That is, to examine whether alerting scores varied across the different flanker displays, we conducted a 2 (no cue and double cue) X 3 (neutral flanker, congruent flanker, and incongruent flanker) repeated meas-

\footnotetext{
${ }^{1}$ Across all participants, 13 to 22 observations were used in each cell to calculate the individual network scores.

${ }^{2}$ Because Fan, McCandliss, Sommer, et al. (2002) did not use the trimmed mean procedure, we did a preliminary analysis to determine whether our results changed when no observations were eliminated. There were no differences in the outcome of our analyses whether we used trimmed mean RTs or mean RTs.
} 
ures ANOVA. Similarly, a 2 (central cue and spatial cue) X 3 (neutral, congruent, and incongruent) repeated measures ANOVA was performed to analyze whether orienting measures varied across flanker conditions. Finally, we conducted a 2 (congruent flanker, incongruent flanker) X 4 (no cue, double cue, central cue, spatial cue) repeated measures ANOVA to determine whether executive control scores varied depending on the type of cue that preceded the flanker display.

We also used Pearson's correlations to examine the relation between cue and flanker conditions for each of the attention network scores. For example, Pearson's $r$ was calculated to determine whether the four executive control measures (no cue executive control, double cue executive control, central cue executive control, and spatial cue executive control) were correlated with one another. The same correlation procedure was used for the three measures of alerting (neutral flanker alerting score, congruent flanker alerting score, and incongruent flanker alerting score) and the three measures of orienting (neutral orienting, congruent orienting, and incongruent orienting). Because the magnitude of the correlation coefficient is a measure of effect size, we defined significant correlations as follows: an $r$ of .01 to .23 is a small effect with negligible practical importance; an $r$ of .24 to .36 is a medium effect with moderate practical importance; and an $r$ of .37 or greater is a large effect with crucial practical importance (Cohen, 1987, as cited in Hojat \& $\mathrm{Xu}, 2004)$.

\section{Results}

The means and standard errors for trimmed mean RTs and overall trimmed mean values were $49.1 \mathrm{~ms}$ for the alerting network, $34.7 \mathrm{~ms}$ for the orienting network, and $110.5 \mathrm{~ms}$ for the executive control network. In terms of mean error rate, the mean values for the alerting, orienting, and executive control networks were $-0.6 \%, 1.2 \%$, and $5.1 \%$, respectively.

\section{ANOVA}

For both trimmed mean RTs and mean accuracy, the 4 (cue condition) x 3 (flanker condition) ANOVA revealed significant main effects for both cue condition [RT, $F(3,1,731)=1888.5$, error rates are presented in Tables 1 and 2, respectively. The

$p<.001$; error rate, $F(3,1,731)=41.7, p<.001]$ and flanker type $[\mathrm{RT}, F(2,1,154)=4689.3, p<.001$; error rate, $F(2,1,154)=455.1, p<.001]$. Additionally, there was a significant Cue X Flanker interaction [RT, $F(6,3,462)=98.2$, $p<.001$; error rate, $F(6,3,462)=35.9, p<.001]$. The nature of the interaction was identical to that reported in Fan, McCandliss, Sommer, et al.'s (2002) original description of the ANT, whereby the flanker interference effect was reduced for both the no cue and spatial cue conditions relative to the double cue and central cue conditions. We will now discuss the Flanker X Cue relationship for each of the three attention measures.

Alerting network The 2 (no cue and double cue) X 3 (neutral flanker, congruent flanker, and incongruent flanker) repeated measures ANOVA revealed an effect of cue and flanker conditions for both trimmed mean RTs and error rates, $F_{S}>$ 20.1, $p<.001$. The cue condition and flanker display interacted with one another to influence both dependent measures, $F_{\mathrm{S}}>356.7, p<0.001$; see Fig. 2. Post hoc comparisons across trimmed mean RTs and error rates revealed that the magnitude of the alerting effect was reduced for incongruent flankers relative to congruent and neutral flankers, $t>4.9, p<.001$. For both mean RTs and error rates, alerting scores were equivalent across congruent and neutral flanker conditions for both dependent variables, $t<0.71, p>.38$.

Orienting network The 2 (central cue and spatial cue) X 3 (neutral, congruent, and incongruent) ANOVA revealed a significant effect of cue condition, $F s>105.4, p<.001$, and flanker types, $F s>376.0, p<.001$, for both trimmed mean RT and error rates. Both of the dependent variables also showed a Cue X Flanker interaction, $F_{s}>57.4, p<$ .001; see Fig. 3. For both trimmed RTs and error rates, orienting scores for incongruent flankers were significantly greater than those for congruent and neutral flankers, $t>$ 4.7, $p<.001$, whereas there was no difference between congruent and neutral flanker conditions, $t<0.8, p>.42$.

Executive control network The 2 (congruent flanker, incongruent flanker) X 4 (no cue, double cue, central cue, spatial cue) ANOVA revealed significant influence of cue and flanker types for both trimmed mean RTs and error rates, $F_{s}>48.4, p<.001$. Again, both dependent variables showed a significant

Table 1 Trimmed mean reaction times and standard error data for cue and flanker conditions

\begin{tabular}{|c|c|c|c|c|c|c|c|c|c|c|}
\hline & \multicolumn{2}{|c|}{ No Cue } & \multicolumn{2}{|c|}{ Central Cue } & \multicolumn{2}{|c|}{ Double Cue } & \multicolumn{2}{|c|}{ Spatial Cue } & \multicolumn{2}{|c|}{ Marginal Mean } \\
\hline & $M$ & $S E$ & $M$ & $S E$ & $M$ & $S E$ & $M$ & $S E$ & $M$ & $S E$ \\
\hline Neutral & 517.1 & 2.6 & 464.0 & 2.4 & 458.5 & 2.3 & 437.0 & 2.6 & 469.2 & 2.2 \\
\hline Congruent & 533.8 & 2.9 & 478.0 & 2.7 & 473.9 & 2.6 & 452.3 & 2.6 & 484.5 & 2.5 \\
\hline Incongruent & 624.4 & 3.5 & 605.7 & 3.2 & 595.5 & 3.2 & 554.4 & 3.2 & 595.0 & 3.1 \\
\hline Marginal Mean & 558.4 & 2.8 & 515.9 & 2.6 & 509.3 & 2.5 & 481.2 & 2.5 & & \\
\hline
\end{tabular}


Table 2 Mean error rates (in percentages) and standard error data for cue and flanker conditions

\begin{tabular}{|c|c|c|c|c|c|c|c|c|c|c|}
\hline & \multicolumn{2}{|c|}{ No Cue } & \multicolumn{2}{|c|}{ Central Cue } & \multicolumn{2}{|c|}{ Double Cue } & \multicolumn{2}{|c|}{ Spatial Cue } & \multicolumn{2}{|c|}{ Marginal Mean } \\
\hline & $M$ & $S E$ & $M$ & $S E$ & $M$ & $S E$ & $M$ & $S E$ & $M$ & $S E$ \\
\hline Neutral & 1.6 & 0.1 & 1.7 & 0.1 & 1.3 & 0.1 & 1.3 & 0.1 & 1.5 & 0.1 \\
\hline Congruent & 1.4 & 0.1 & 1.2 & 0.1 & 1.2 & 0.1 & 0.9 & 0.1 & 1.7 & 0.1 \\
\hline Incongruent & 5.5 & 0.3 & 8.6 & 0.4 & 7.8 & 0.4 & 5.5 & 0.3 & 6.8 & 0.3 \\
\hline Marginal Mean & 2.8 & 0.1 & 3.8 & 0.2 & 3.4 & 0.2 & 2.6 & 0.1 & & \\
\hline
\end{tabular}

interaction between cue and flanker, $F s>39.5, p<.001$; see Fig. 4.

For trimmed mean RTs, the executive control measures across all four cue types were significantly different from one another, even after Bonferroni corrections were applied, $t>3.3$, $p<.001$. However, the flanker interference effect was reduced when the flanker display was preceded by no cue or by a spatial cue relative to double and central cues (see Fig. 4). The flanker effect for mean error rates was also reduced for both the no cue and spatial cue conditions relative to the double cue and central cue conditions. Although the magnitude of the flanker interference effect in the no cue/spatial cue condition relative to that in the double cue and central cue, $t>4.3, p<$ .001 , conditions, there was no difference in the flanker effect for mean error rates across the no cue and spatial cue conditions, $t(576)=0.17, p=.87$, and the double cue and center cue conditions, $t(576)=0.77, p=.44$.

\section{Correlational analyses}

Before analyzing the intranetwork correlations for each measure of attention, we conducted a between-network correlational analysis to allow for a comparison of our findings

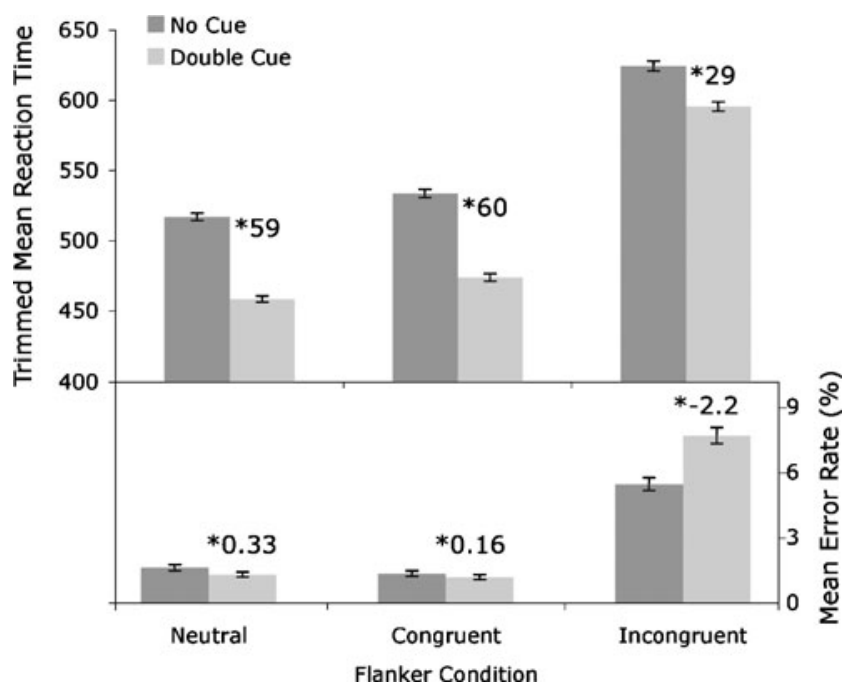

Fig. 2 Trimmed mean reaction times (RTs) and errors for no cue and double cue trials for each of the three flanker conditions. The asterisked numerical values represent the mean alerting score for each flanker condition. Error bars represent the standard errors of the means with those of Fan, McCandliss, Sommer, et al. (2002). For trimmed mean RTs, none of the between-network correlations was significant (alerting vs. orienting, $r=.07$; alerting vs. executive control, $r=.01$; and orienting vs. executive control, $r=-.04)$. These between-network correlations are similar to those reported by Fan, McCandliss, Sommer, et al. (2002). For mean error rates, all correlations were significant. The largest correlation was between alerting and executive control $(r=-.35)$, followed by orienting and executive control $(r=.33)$. Alerting and orienting had the smallest correlation $(r=-.23)$.

The alerting and orienting intranetwork correlation matrices are found in Table 3, and the executive control intranetwork correlations are found in Table 4.

Alerting For alerting trimmed mean measures, there were small correlations between neutral and incongruent flankers $(r=.11)$ and medium correlations between neutral and congruent flankers $(r=.29)$. There was no relationship between trimmed RT measures for congruent and incongruent flankers $(p=.15)$.

Error rates revealed a small, significant correlation between neutral and incongruent flankers $(r=.09, p<.05)$. All other correlations failed to reach significance $(p>.30)$.

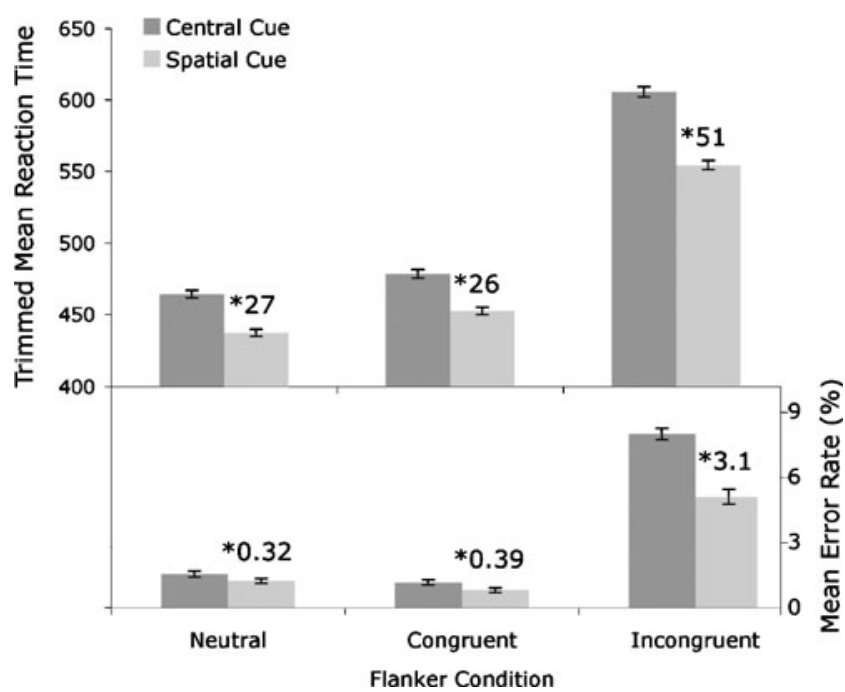

Fig. 3 Trimmed mean reaction times (RTs) and errors for central cue and spatial cue trials for each of the three flanker conditions. The asterisked numerical values represent the mean orienting score for each flanker condition. Error bars represent the standard errors of the means 


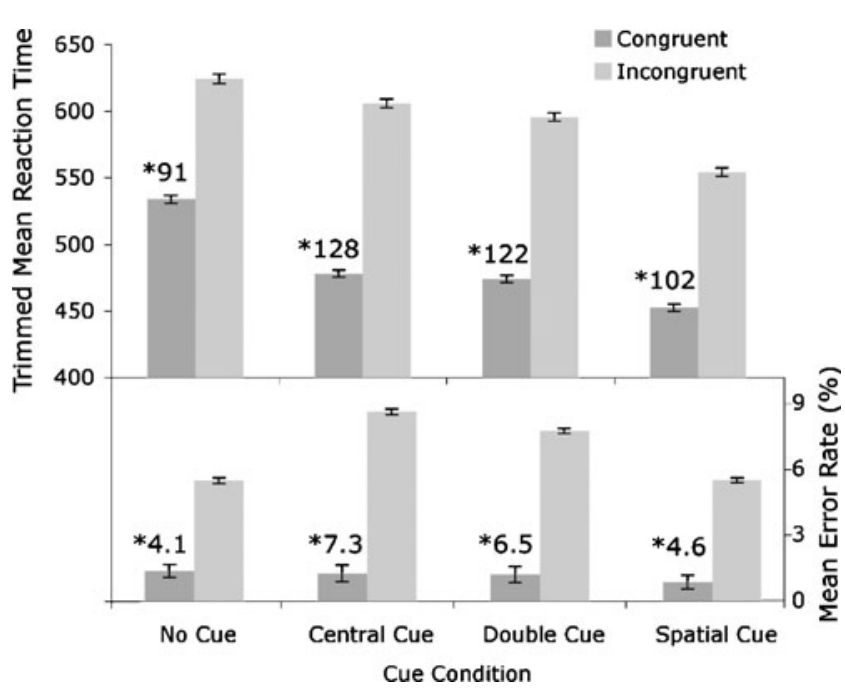

Fig. 4 Trimmed mean RTs and errors for congruent and incongruent trials for each of the four cue conditions. The asterisked numerical values represent the trimmed mean executive function score for each cue condition. Error bars represent the standard errors of the means

Orienting All orienting trimmed RT scores across the different flanker types were significantly correlated with one another. There was a medium correlation between neutral and congruent flankers $(r=.23)$ and between congruent and incongruent flankers $(r=.27)$. The correlation between neutral and incongruent flankers was small $(r=.16)$

The only significant correlation for orienting error rates was between neutral and congruent flankers $(r=.10, p<.05)$. All other correlations failed to reach significance $(p>.36)$.

Executive control All executive control measures were moderately correlated with one another for both trimmed RTs and error rates $(r>.37, p<.001)$. Of the three attention networks, the executive control measure had the highest correlations among the separable executive control factors (Pearson's $r$ ranging from $.42-.54$ for trimmed RT, and $.37-.52$ for error rates).

\section{Discussion}

In the present study, we sought to analyze the ANT's intranetwork variability for each of the three components of attention. The efficiency of alerting, orienting, and executive control is typically calculated using a series of orthogonal contrasts, in which RT measures and error rates are averaged across several cue-flanker conditions. This procedure assumes that the collapsed components of each network are all providing reliable measures of the same process. These assumptions are somewhat perplexing, since numerous ANT studies have reported Flanker X Cue interactions (i.e., Costa, Hernández, Sebastián-Gallés, \&
2008; Fan, Byrne, et al., 2007; Johnson et al., 2008; Oberlin, Alford, \& Marrocco, 2005; Redick \& Engle, 2006). In the present study, we used ANOVAs and correlational procedures to examine the relation between the four executive control measures (no cue executive control, double cue executive control, central cue executive control, and spatial cue executive control), the three measures of alerting (alerting scores for neutral flankers, congruent flankers, and incongruent flankers) and the three measures of orienting (orienting for neutral, congruent, and incongruent flankers).

We showed significant differences across cue-flanker conditions in the ANT for both trimmed mean RTs and mean error rates. Alerting and orienting scores for incongruent flankers were significantly different than those obtained for neutral and congruent flankers. Interestingly, incongruent flankers had contrasting effects across alerting and orienting networks: The efficiency of the ANT's alerting measure was reduced for incongruent flankers, whereas the efficiency of the ANT's orienting measure was enhanced for incongruent flankers.

The flanker congruency effect varied as a function of cue type. Reduced flanker effects were found when the arrow display was preceded by no cue or a spatial cue as compared with when it was preceded by a central or double cue. This effect is similar to that obtained by Fan, McCandliss, Sommer, et al. (2002), whereby the flanker effect was enhanced following a double or central cue versus no cue or a spatial cue. Our results suggest that researchers should avoid collapsing across different cue and flanker conditions to get single measurements of the three attention networks.

The observed patterns of data raise several important issues. Why is the flanker effect enhanced at the no cue and spatial cue conditions, but reduced at the central and double cue conditions? These findings may be the result of global versus local processing strategies. Successful completion of the ANT requires participants to focus attention on local

Table 3 Intranetwork correlation matrix. Each of the panels above the diagonal (upper right) shows the correlations among the trimmed mean measures for both the alerting and orienting networks. The panels below the diagonal (bottom left) show the correlations between the mean error scores for alerting and orienting networks

\begin{tabular}{llll}
\hline & Neutral & Congruent & Incongruent \\
\hline Neutral & Alerting & $.29^{* *}$ & $.11^{* *}$ \\
Congruent & -.04 & Alerting & .06 \\
Incongruent & $.09^{*}$ & -.03 & Altering \\
Neutral & Orienting & $.23^{* *}$ & $.16^{* *}$ \\
Congruent & $.10^{*}$ & Orienting & $.27^{* *}$ \\
Incongruent & .04 & -.03 & Orienting \\
\hline
\end{tabular}

$* p<.05 . * * p<.001$ 
Table 4 Intranetwork correlation matrix. Each of the panels above the diagonal (upper right) shows the correlations among the trimmed mean executive function measures. The panels below the diagonal (bottom left) show the correlations across the mean error scores for the four executive function measures

\begin{tabular}{lllll}
\hline & No Cues & Central Cues & Double Cues & Spatial Cues \\
\hline No Cues & & $.47^{* *}$ & $.41^{* *}$ & $.44^{* *}$ \\
Central Cues & $.41^{* *}$ & $.52^{* *}$ & $.51^{* *}$ & $.54^{* *}$ \\
\cline { 5 - 5 } Double Cues & $.41^{* *}$ & $.48^{* *}$ & $.51^{* *}$ & $.48^{* *}$ \\
Spatial Cues & $.37^{* *}$ & & - \\
\hline
\end{tabular}

$* * p<.001$

aspects of the target display; therefore, while waiting for the cue or target to appear, an efficient strategy would be to focus attention on the fixation point rather than to spread attentional resources across both target locations. When a spatial cue appears, participants simply move their attention to the precise location of the upcoming target. Because attentional resources are already focused on the location of the target arrow when the flanker display appears, cognitive demands are decreased, and less executive control is needed to respond. On the other hand, when the double cue appears, attention is diffused between two locations, making it more difficult to identify target characteristics and ignore irrelevant flanker stimuli. As a result, flanker congruency effects are larger in the double cue condition, resulting in lower measures of executive control.

Although this global/local explanation appears to explain the differences in executive control between spatial and double cue conditions, it is less obvious how this conceptualization can be applied to the reduced flanker effect found in the no cue condition relative to the central cue condition. Based on this global/local hypothesis, one would expect equivalent flanker effects for no cue and central cue trials, since both cue conditions encourage participants to focus their attention on the fixation point. The key difference between these two cue conditions lies in the processing strategy evoked by the participant after the offset of the cue but before the onset of the target. Recall that although the participants are waiting for the cue or target to appear, attention is focused on the fixation point. Therefore, when no cue precedes the onset of the target display, attention is still in a focused state, making it easier to identify local characteristics of the target and to ignore distracting information from the flanker stimuli.

On the other hand, the appearance of a central cue provides participants with relevant temporal information (i.e., the target is about to appear) but no spatial information (i.e., where the target is about to appear). Given the lack of spatial information, attention may spread to both target locations in anticipation of the upcoming target event, requiring more executive control to make the appropriate response when the target appears. This difference in the breadth of attentional resources can explain why the flanker effect is reduced in both the no cue and spatial cue conditions relative to the double cue and central cue conditions.

Fan, McCandliss, Sommer, et al. (2002) explained this Cue X Flanker interaction differently. According to these researchers, participants need more time to respond to targets when no cue is presented, as illustrated by longer RTs in the no cue condition. Because participants need more time to respond, executive control processes have additional time to resolve any perceptual conflict in the target display. Neither the present study nor that by Fan, McCandliss, Sommer, et al. (2002) set out to specifically investigate the Cue X Flanker interaction; therefore, both explanations are purely speculative. Nonetheless, both theories provide a series of testable hypotheses that can be used to investigate measures of executive control.

Another issue raised in the present study was why alerting and orienting effects differ across congruent/neutral versus incongruent flanker types. The efficiency of the ANT's alerting measure was reduced for incongruent flankers, whereas the efficiency of the ANT's orienting measure was enhanced for incongruent flankers. The effect of alerting was seen as longer RTs (and more errors) for no cue trials relative to double cue trials. The reduced flanker effect for the no cue condition would enhance conflict resolution, which would be particularly advantageous when distracting flanker items surround the target arrow (i.e., incongruent trials). Similarly, the larger flanker effect for double cues would impair conflict resolution, which would be particularly disadvantageous on incongruent trials. Therefore, RTs and error rates to incongruent flanker types should be decreased in the no cue condition and increased in the double cue condition, which will result in smaller alerting scores for incongruent flankers than to congruent/ neutral flanker types.

On the other hand, the effect of orienting is characterized by shorter RTs (and fewer errors) in the spatial cue condition than in the central cue condition. The flanker effect is larger for central cues and smaller for spatial cues. We would therefore expect response efficiency to be reduced (i.e., larger RTs and error rates) in the central cue 
Table 5 Reliability measures (and confidence intervals) from MacLeod et al. (2010) and from related simulation using the current data set and cell sizes

\begin{tabular}{llllll}
\hline & \multicolumn{2}{l}{ Current Data Set } & & & \multicolumn{2}{l}{ MacLeod et al. (2010) } \\
\cline { 2 - 3 } \cline { 5 - 6 } & Reaction Time & Error & & Reaction Time & Error \\
\hline Alerting & $0.12(0.04,0.20)$ & $0.02(-0.06,0.10)$ & & $0.20(0.14,0.27)$ & $0.06(0.01,0.12)$ \\
Orienting & $0.13(0.05,0.21)$ & $0.00(-0.09,0.08)$ & & $0.32(0.26,0.38)$ & $0.14(0.07,0.21)$ \\
Executive Function & $0.48(0.41,0.54)$ & $0.40(0.33,0.46)$ & & $0.65(0.61,0.70)$ & $0.71(0.67,0.76)$ \\
\hline
\end{tabular}

condition and enhanced (i.e., smaller RTs and error rates) in the spatial cue condition. This would explain why orienting scores were increased for incongruent trials, when enhanced conflict resolution would be particularly advantageous. Again, these explanations are purely speculative, and more research is needed to determine the validity of these statements. We encourage future researchers to examine the potential effects of global/local processing on attention network scores.

In addition to analyzing mean differences across cueflanker combinations, we also conducted correlational analyses to determine the extent to which individual measures of the same network were correlated with one another. These correlations were surprisingly low, considering the ANT's assumption that the collapsed factors are all measuring the same attentional process. Using Cohen's (1987, as cited in Hojat \& Xu, 2004) guidelines for effect size, the three alerting measures and the three orienting measures showed low to low-medium correlations (alerting $r$ range, -.03-.29; orienting $r$ range, -.03-.28). Fortunately, the four different executive control measures were moderately correlated (executive control $r$ range: .37-.54).

As a point of comparison for these correlations, we examined the split-half reliabilities produced by MacLeod et al. (2010). MacLeod et al. examined the psychometric characteristics of the ANT using data collected from 1,141 participants in 15 unique studies that administered the ANT to healthy individuals. ${ }^{3}$ These researchers calculated splithalf reliabilities for alerting, orienting, and executive control networks. To do this, they used a permutation method whereby the correlation within each network was calculated for the mean of 10,000 split-half estimates (for further information on the permutation method, see MacLeod et al., 2010; see Table 5, left column, for their values). A comparison of Tables 3 and 4 with Table 5 indicate that a number of intranetwork correlations are below the confidence intervals established using this split-half reliability.

Although this is disconcerting, the data may be misleading since the MacLeod et al. (2010) data were based on more observations per cell. Specifically, for alerting and orienting, there are 72 relevant trials resulting in 36 potential observations for each reliability measure. For the

\footnotetext{
${ }^{3}$ The data presented here form a subset of the data used in the MacLeod et al. (2010) analyses.
}

executive function network, there were 96 relevant trials resulting in 48 potential observations for each reliability measure. ${ }^{4}$ The correlations computed in the present manuscript were based on only 24 observations. Additionally, we used a trimmed mean procedure, which further reduced the number of potential observations to 20 . In order to provide a relevant point of comparison, the split-half reliabilities were recomputed using these parameters. That is, two sets of 24 observations were selected from the potential set of 72 observations (96 for executive function), and these data were trimmed and error rates computed. These two sets of 24 observations were then correlated across participants 10,000 times, and the resulting estimates of reliability were computed (see Table 5, left side). With this comparison, all of the intranetwork correlations are within the confidence intervals, or above them. The fact that some intranetwork correlations are better than the average reliability indicates that some cells in the design provide a more reliable estimate of the cognitive construct than others. This finding directly undermines the assumption that one can simply average across the levels of the other factor (cue type or flanker type) to provide an orthogonal measure. It is also interesting to compare the reliability estimates derived from the present set of observations with those derived from the MacLeod et al. (2010) study. Specifically, the present analysis equates the number of observations used for each network, whereas the previous analysis did not. This change drastically reduces the reliability of all of the networks, but does so most markedly for the executive function network.

On a more positive note, although the intranetwork correlations reported in the present study were lower than we expected, they were still larger than the observed betweennetwork correlations. However, just because the intranetwork correlations were larger than the between-network correlations does not mean that researchers should be satisfied with these measures. If two unreliable measures are correlated with each other, then the correlation between them should be small. Therefore, the lack of a correlation between networks may arise because the measures are simply not reliable. It is important for future researchers to examine relations both between and within

\footnotetext{
${ }^{4}$ It is interesting to note that there are more observations for the executive function network in the MacLeod et al. data analysis, and that this measure provided the most reliable index.
} 
the ANT's measures of alerting, orienting, and executive control in order to determine the most appropriate way to measure these three components of attention.

Importantly, our results do not speak to the independence of the networks themselves, but rather point to the interdependence of the ANT's cue-flanker conditions. That is, the alerting, orienting, and executive control networks may all contribute to the behavioral measures obtained by the ANT. This is consistent with recent research showing that the three attentional subsystems are able to work together to influence behavior. Research has shown that the alerting network inhibits executive control, whereas the orienting network enhances executive control (Callejas, Lupiáñez, Funes, et al., 2005; Callejas, Lupiáñez, \& Tudela, 2004; Fan, Gu, et al., 2009) and the alerting network modulates orienting effects (Fuentes \& Campoy, 2008). Furthermore, there is substantial functional overlap across the different neural networks (Fan et al., 2005). These findings, coupled with those from the present study, suggest that these anatomically isolated components of attention work in concert to produce interactive cognitive processing. Future work on attentional networks needs to focus on quantifying the relevant interactions. Two notable alternatives to the ANT - the ANT-i (Callejas, Lupiáñez, Funes, et al., 2005) and the ANT-R (Fan et al., 2009) -have been developed to specifically examine interactions between networks. These modified versions of the ANT appear to provide more accurate assessments of the relationships between and within the three networks of attention.

Although there is little debate that attention is composed of several subsystems, the results of the present study suggest that the ANT is not the most appropriate way to independently assess the efficiency of each attentional component. This finding is particularly important, given the fact that the ANT is used so pervasively throughout clinical, neurological, and experimental psychology. Many studies have used the ANT to demonstrate specific attentional deficits in special populations, or to investigate individual differences in attention networks in the normal population. In order for such interpretations to have meaning, it is critical that the ANT provide reliable and valid measures of the intended attentional constructs. In order to take any conclusions on network-specific phenomena seriously, more research on the psychometric properties of the ANT is required. It is essential to determine whether the low reliability of attention measures provided by the ANT is due to the task itself or to the high variability of the efficiency of attention networks both between and within individuals.

Author Note We thank Juan Lupiáñez and two anonymous reviewers for their helpful comments and suggestions. This research forms the fourth chapter of the PhD thesis for M.M.M., who was supported by NSERC through a PGS-D. D.I.S. was supported by an NSERC discovery grant and a Premier's Research Excellence Award from the Ministry of Economic Development and Trade.

\section{References}

Bellgrove, M. A., Hawi, Z., Gill, M., \& Robertson, I. H. (2006). The cognitive genetics of attention deficit hyperactivity disorder (ADHD): Sustained attention as a candidate phenotype. Cortex, 42, 838-845.

Brainard, D. H. (1997). The Psychophysics toolbox. Spatial Vision, $10,433-436$.

Bush, G., Luu, P., \& Posner, M. I. (2000). Cognitive and emotional influences in the anterior cingulate cortex. Trends in Cognitive Science, 4, 215-222.

Callejas, A., Lupiáñez, J., Funes, M. J., \& Tudela, P. (2005). Modulations among the alerting, orienting, executive control networks. Experimental Brain Research, 167, 27-37.

Callejas, A., Lupiáñez, J., \& Tudela, P. (2004). The three attentional networks: On their independence and interactions. Brain and Cognition, 54, 225-227.

Cohen, J. (1987). Statistical power analysis for behavioral sciences. Hillsdale, NJ: Erlbaum.

Corbetta, A. B. (1998). Frontoparietal cortical networks for directing attention and the eye to visual locations: Identical, independent, or overlapping neural systems? Proceedings of the National Academy of Science, 95, 831-838.

Corbetta, A. B., Kincade, J. M., Ollinger, J. M., McAvoy, M. P., \& Shulman, G. (2000). Voluntary orienting is dissociated from target detection in human posterior parietal cortex. Nature Neuroscience, 3, 292-297.

Costa, A., Hernández, M., \& Sebastián-Gallés, N. (2008). Bilingualism aids conflict resolution: Evidence from the ANT task. Cognition, 106, 59-86.

Coull, J. T., Frith, C. D., Frackowiak, R. S., \& Grasby, P. M. (1996). A fronto-parietal network for rapid visual information processing: A PET study of sustained attention and working memory. Neuropsychologia, 34, 1085-1095.

Coull, J. T., Nobre, A. C., \& Frith, C. D. (2001). The noradrenergic $\alpha 2$ agonist clonidine modulates behavioural and neuroanatomical correlates of human attentional orienting and alerting. Cerebral Cortex, 11, 73-84.

Davidson, M. C., Cutrell, E. B., \& Marrocco, R. T. (1999). Scopolamine slows the orienting of attention in primates to cued visual targets. Psychopharmacology, 142, 1-8.

Diamond, A., Briand, L., Fossella, J., \& Gehlbach, L. (2004). Genetic and neurochemical modulation of prefrontal cognitive functions in children. The American Journal of Psychiatry, 161, 125-132.

Erceg-Hurn, D., \& Mirosevich, V. M. (2008). Modern robust statistical methods: An easy way to maximize the accuracy and power of your research. The American Psychologist, 63, 591-601.

Etkin, A., Egner, T., Peraza, D. M., Kandel, E. R., \& Hirsch, J. (2006). Resolving emotional conflict: A role for the rostral anterior cingulate cortex in modulating activity of the amygdala. Neuron, 51, 871-882.

Fan, J., Byrne, J., Worden, M. S., Guise, K., McCandliss, B. D., Fossella, J., \& Posner, M. I. (2007). The relation of brain oscillations to attentional networks. The Journal of Neuroscience, 27, 6197-6206.

Fan, J., Gu, X., Guise, K. G., Liu, X., Fossella, J., Wang, H., \& Posner, M. I. (2009). Testing the behavioural interaction and integration of attentional networks. Brain and Cognition, 70, 209-220.

Fan, J., McCandliss, B. D., Fossella, J., Flombaum, J. I., \& Posner, M. I. (2005). The activation of attentional networks. Neuroimage, 26, 471-479.

Fan, J., McCandliss, B. D., Sommer, T., Raz, A., \& Posner, M. I. (2002). Testing the efficiency and independence of attentional networks. Journal of Cognitive Neuroscience, 14, 340-347.

Fossella, J. A., Sommer, T., Fan, J., Wu, Y., Swanson, J. M., Pfaff, D. W., \& Posner M. I. (2002). Assessing the molecular genetics of attention networks. BMC Neuroscience, 3, 14. 
Fuentes, L. J., \& Campoy, G. (2008). The time course of alerting effect over orienting in the attention network test. Experimental Brain Research, 185, 667-672.

Greenwood, P. M., Fossella, J. A., \& Parasuraman, R. (2005). Specificity of the effect of a nicotinic receptor polymorphism on individual differences in visuospatial attention. Journal of Cognitive Neuroscience, 17, 1611-1620.

Hojat, M., \& Xu, G. (2004). A visitor's guide to effect sizes: Statistical significance versus practical (clinical) importance of research findings. Advances in Health Sciences Education, 9, 241-249.

Husain, G., Thompson, W. F., \& Schellenberg, E. G. (2002). Effects of musical tempo and mode on arousal, mood, and spatial abilities. Music Perception, 20, 151-171.

Johnson, K. A., Robertson, I. H., Barry, E., Mulligan, A., Dáibhis, A., Daly, M., Bellgoove, M. A. (2008). Impaired conflict resolution and alerting in children with ADHD: Evidence from the attention network task (ANT). Journal of Child Psychology and Psychiatry, 49, 1339-1347.

MacDonald, A. W., Cohen, J. D., Stenger, V. A., \& Carter, C. S. (2000). Dissociating the role of the dorsolateral prefrontal and anterior cingulate cortex in cognitive control. Science, 288, 1835-1838.

MacLeod, J., Lawrence M. A., McConnell, M. M., Eskes, G. A., Klein, R. M., \& Shore, D. I. (2010). Appraising the ANT: Psychometric and Theoretical Considerations of the Attention Network Test. Neuropsychology, 24, 637-651.

McConnell, M. M., \& Shore, D. I. (in prep). A time to work and a time to play: Temporal variations in measures of attention. Manuscript in preparation.
McConnell, M. M., \& Shore, D. I. (submitted). The effects of shifts to and from daylight savings time on attentional network. Manuscript submitted for publication.

McConnell, M. M., \& Shore, D. I. (in press). Upbeat and happy: Arousal as an important factor in studying attention. Cognition \& Emotion.

Oberlin, B. G., Alford, J. L., \& Marrocco, R. T. (2005). Normal attention orienting but abnormal stimulus alerting and conflict effect in combined subtype of ADHD. Behavioural Brain Research, 165, 1-11.

Parasuraman, R., Greenwood, P. M., Haxby, J. B., \& Grady, C. L. (1992). Visuospatial attention in dementia of the Alzheimer type. Brain, 115, 711-733.

Parasuraman, R., Greenwood, P. M., Kumar, R., \& Fossella, J. (2005). Beyond heritability: Neurotransmitter genes differentially modulate visuospatial attention and working memory. Psychological Science, 16, 200-207.

Posner, M. I., \& Fan, J. (2004). Attention as an organ system. In J. R. Pomerantz \& M. C. Crai (Eds.), Topics in integrative neuroscience: From cells to cognition (pp. 31-62). Cambridge: Cambridge University Press.

Posner, M. I., \& Petersen, S. E. (1990). The attention system of the human brain. Annual Review of Neuroscience, 13, 25-42.

Redick, T. S., \& Engle, R. W. (2006). Working memory capacity and attention network test performance. Applied Cognitive Psychology, 20, 713-721.

Sturm, W., \& Willmes, K. (2001). On the functional neuroanatomy of intrinsic and phasic alertness. Neuroimage, 14, 76-84. 